\title{
79 Die Biomechanik des Laufens
}

(c) Springer-Verlag GmbH Deutschland, ein Teil von Springer Nature 2018

D. Mathias, Fit und gesund von 1 bis Hundert

https://doi.org/10.1007/978-3-662-56307-6_79

Barfuß oder mit dünnem Schuhwerk laufende Menschen setzen eher mit dem Vor- oder Mittelfuß auf. Erst durch die heute verbreiteten gepolsterten Laufschuhe wurden viele Jogger zu Fersenläufern und sind dadurch vermehrt Überlastungsschäden ausgesetzt (Liebermann et al. 2010; Almeida et al. 2015; van der Worp et al. 2016). Durch die Polsterung sind die Kollisionskräfte auf den Vorfuß geringer als jene auf die Ferse.

26 Knochen bilden das Grundgerüst des Fußes und insgesamt 45 Muskeln pro Bein sind notwendig, um Haltearbeiten und die Fortbewegung in der aufrechten Position zu gewährleisten.

Bei der Fortbewegung muss der Fuß pro Schritt zwei Kraftspitzen auffangen, die erste etwa 20-30 Millisekunden (ms) nach Bodenkontakt als Abfederungsspitze und die zweite nach weiteren $70 \mathrm{~ms}$ als $\mathrm{Ab}$ drückkraft, die den Fuß in Laufrichtung wieder abheben lässt. In der Abstoßphase sind die Knochen- und Gelenkbelastungen höher als beim Fußaufsetzen. Während der Landephase berührt der Fuß im Normalfall zuerst mit seiner vorderen Außenkante den Boden, rollt dann nach innen, um in einer sehr kurzen Standphase eine Pronationsstellung einzunehmen.

Die Belastungen der unteren Extremitäten sind hierbei enorm. Je nach Laufgeschwindigkeit lastet auf den Sprunggelenken bei jedem Schritt für 20$40 \mathrm{~ms}$ das 2,2- bis 4,8-Fache des Körpergewichts. Im Mittel addieren sich die Gewichtsbelastungen für jeden Fuß bei einer $75 \mathrm{~kg}$ schweren Person und einem Laufkilometer (1200 Schritte) auf rund 160 Tonnen über etwa 18 Sekunden.

Wer also neben den alltäglichen Gehstrecken $20 \mathrm{~km}$ pro Woche joggt und dieses Pensum 40 Jahre aufrecht hält, belastet damit in dieser Zeit jedes Sprunggelenk, verteilt über etwa 200 Stunden, zusätzlich mit ca. 6,5 Millionen Tonnen. Dieser Belastung steht eine erstaunliche Anpassungsfähigkeit der biologischen Gewebe gegenüber. Besonders ein muskulärer Trainingseffekt ist schon wenige Wochen nach Übungsbeginn bzw. nach Steigerung des Trainingsumfangs messbar ( $\triangleright$ Kap. 86). Nicht ganz so effektiv verhält sich bei diesen Anpassungsprozessen das Bindegewebe. Strukturen wie Knorpel, Sehnen, Bänder und Kapseln brauchen wegen ihrer geringeren Stoffwechselaktivitäten hierfür bis zu 12 Monate.

Supination
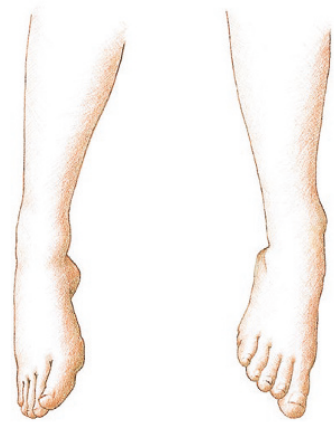

Pronation

- Abb. 79.1 Fußhaltungen bei Belastung (übertriebene Darstellung) 